\title{
Increase in Salmonella enterica serovar Paratyphi A infections in Phnom Penh, Cambodia, January 2011 to August 2013
}

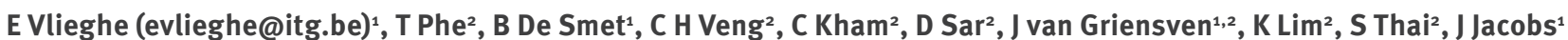

1. Institute of Tropical Medicine, Antwerp, Belgium

2. Sihanouk Hospital Centre of HOPE, Phnom Penh, Cambodia

We report an increased number of Salmonella enterica Paratyphi $A$ infections in adults in Cambodia. Between January 2011 and August 2013, 71 S. Paratyphi A isolates were recovered from blood cultures, representing a 44 -fold increase compared to July 2007 to December 2010, while monthly numbers of cultures did not change. Infections with $S$. Typhi increased two-fold in the same period. Most cases came from the capital Phnom Penh. These findings warrant epidemiological investigation to support public health measures.

Between 1 January 2011 and 31 August 2013, there has been a marked increase of Salmonella enterica serovar Paratyphi A cases diagnosed from blood cultures in Cambodian citizens, particularly in 2013, and this was reflected in an increased recovery of this pathogen from European travellers returning from Cambodia between January and August 2013 [1]. Here, we report preliminary surveillance data from Cambodia.

\section{Background}

S. enterica is an important pathogen in many low and middle income countries [2]. The serovars S. Typhi and Paratyphi cause enteric fever (i.e. typhoid and paratyphoid fever respectively), and are particularly prevalent in south and southeast Asia. Treatment has become challenging because of emerging antibiotic resistance to first-line antibiotics such as chloramphenicol, ampicillin, sulphamethoxazole-trimethoprim (SMX-TMP) and more recently fluoroquinolones [3]. Over the past two decades, S. Paratyphi A has become increasingly prevalent in Asia, causing between $15 \%$ (Pakistan, Indonesia) and $64 \%$ (southeast China) of enteric fever cases in these countries [4]. Typhoid and paratyphoid fever are also endemic in Cambodia [5-7]. Nationwide surveillance of incidence and antibiotic resistance patterns is, however, largely lacking due to the country's very limited microbiology laboratory infrastructure [8]. Since July 2007, the Cambodian non-governmental (NGO) hospital Sihanouk Hospital Centre of HOPE (SHCH) in Phnom Penh, and the Institute of Tropical
Medicine, Antwerp, Belgium co-organise surveillance of bloodstream infections in Cambodian adults attending $\mathrm{SHCH}$. The 30-bed adults' hospital and its associated clinics provide over 135,000 outpatient visits and about 1,000 hospitalisations per year of patients from across Cambodia. Of 6,881 blood cultures drawn between July 2007 and December 2010, we recorded two patients infected with S. Paratyphi A [9].

\section{Laboratory procedures for surveillance of bloodstream infections}

From all patients presenting at SHCH with signs of the Systemic Inflammatory Response Syndrome (SIRS) [10], $2 \times 10 \mathrm{ml}$ of venous blood are sampled by separate venipuncture with registration of demographic and clinical data (i.e. sex, age, province and district of residence, co-morbidity, prior use of antibiotics, type and duration of symptoms, presumed focus of infection, hospitalisation status). Blood is cultured in BacT/ALERT culture bottles (bioMérieux, Marcy l'Etoile, France) which are incubated at $35^{\circ} \mathrm{C}$ for seven days and checked once daily for growth by visual inspection of the chromogenic growth indicator. When positive, blood cultures are Gram stained and -in case Gramnegative rods are present- inoculated on MacConkey agar and $5 \%$ sheep blood agar (BIO-RAD, Hercules, United States). In addition, blood culture vials with no indication of growth after three days of incubation are subcultured on sheep blood agar. As part of standard patient care, isolates are identified by conventional methods and assessed for antibiotic susceptibility by disk diffusion (Neo-SensitabsTM, Rosco Diagnostica, Taastrup, Denmark) according to the Clinical Laboratory Standards Institute [11]. Serotyping is carried out by slide agglutination with commercial antisera according to the Kauffmann-White scheme [12].

In this report, 'paratyphoid fever' and 'typhoid fever' cases were defined as a patient with culture-confirmed bloodstream infection due to S. Paratyphi A or S. Typhi respectively. Patients with blood cultures that did not 


\section{FIGURE 1}

Salmonella Paratyphi A and Salmonella Typhi infections diagnosed at Sihanouk Hospital Centre of HOPE, Phnom Penh, Cambodia, January 2011-August 2013 ( $\mathrm{n}=102$ )

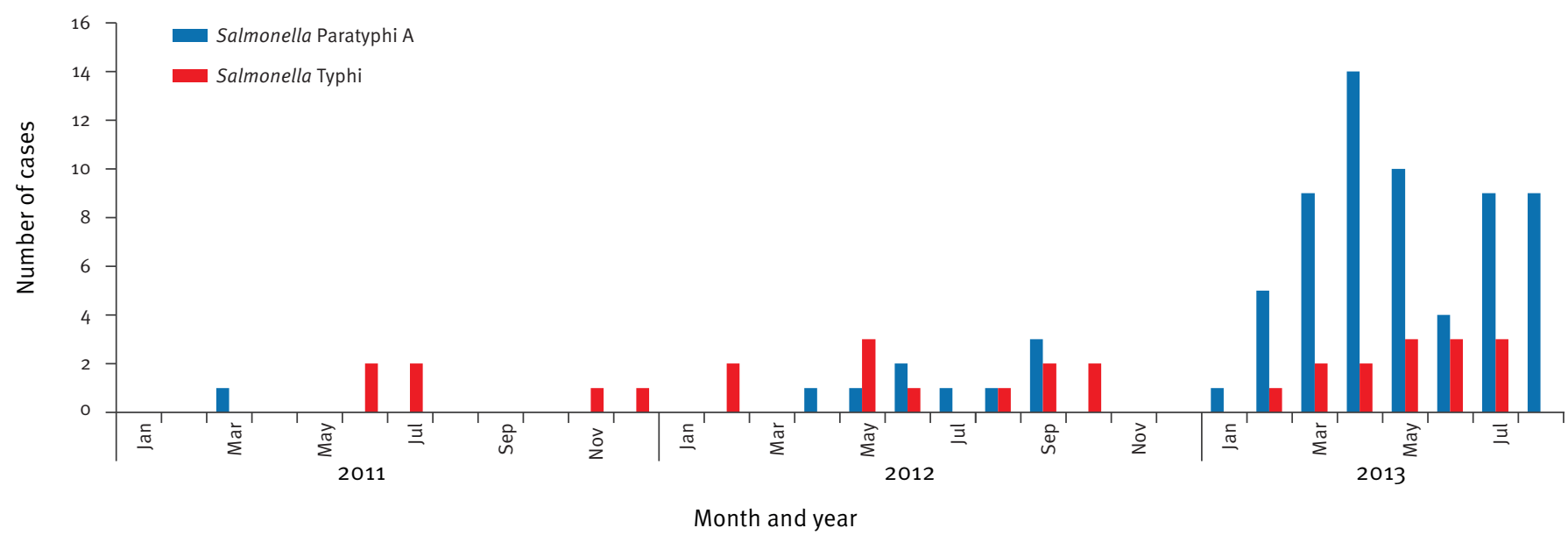

\section{FIGURE 2}

Geographical origin of Salmonella Paratyphi A cases diagnosed at Sihanouk Hospital Centre of HOPE, Phnom Penh, Cambodia, January 2011-August 2013 (n=71)

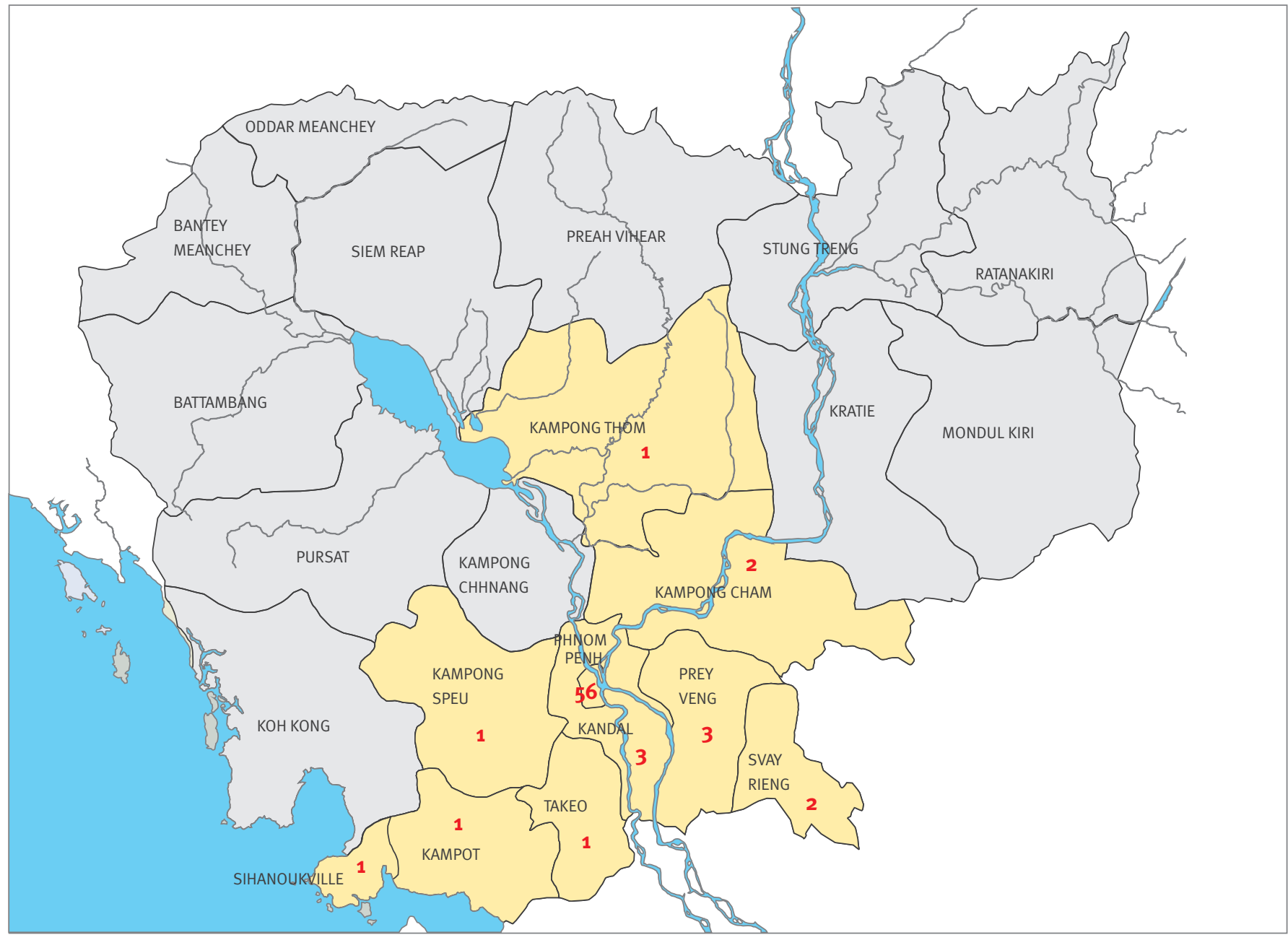




\section{FIGURE 3}

Distribution of Salmonella Paratyphi A cases living in Phnom Penh, diagnosed at Sihanouk Hospital Centre of HOPE, Phnom Penh, Cambodia, January 2011-August $2013\left(n=56^{\mathrm{a}}\right)$

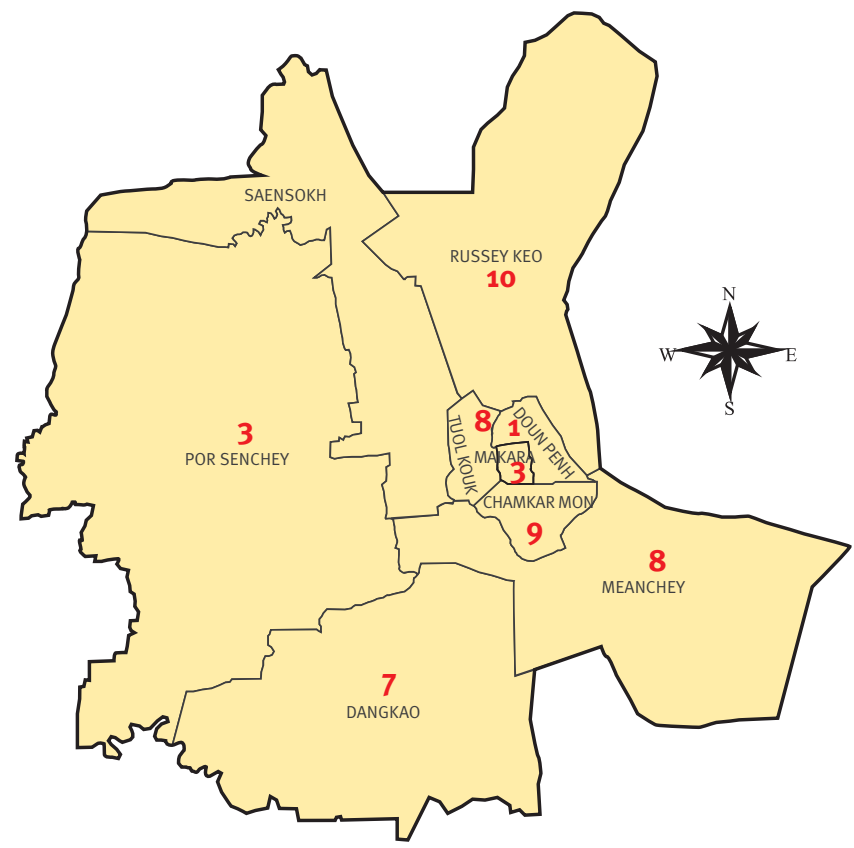

${ }^{a}$ District unknown for seven cases.

grow after complete work-up or that revealed pathogens other than 'S. Typhi/Paratyphi A' were not considered 'cases' for the purpose of this investigation.

Ethical approval was granted by the review boards at Institute of Tropical Medicine, Antwerp, the University Hospital Antwerp and the National Ethics Committee for Health Research, Phnom Penh, Cambodia respectively.

\section{Surveillance of blood cultures, 1 January 2011 to 31 August 2013}

Between 1 January 2011 and 31 August 2013, 102 cases of enteric fever were diagnosed in Cambodian citizens i.e. 71 with paratyphoid fever and 31 with typhoid fever. Seven cases were recorded in 2011, 20 in 2012 and 75 in the first eight months of 2013. As shown in Figure 1, paratyphoid fever cases were observed more frequently from April 2012 and numbers increased further from March 2013 onwards. Typhoid fever cases increased as well but to a lesser extent.

Forty-seven per cent (i.e. 35 of 71 (49.3\%) paratyphoid cases and 13/31 (41.9\%) typhoid cases) were female, with a median age of 24 years (range $7-54$ years); ages of paratyphoid and typhoid cases were not significantly different.

Out of 71 paratyphoid fever cases, 56 lived in the greater Phnom Penh area (Figure 2), particularly in the following districts: Russey Keo $(n=10)$, Chamkar Mon $(n=9)$, Tuol Kouk $(n=8)$, Mean Chey $(n=8)$, Dangkao $(n=7)$, Prampir Makara $(n=3)$, Por Senchey $(n=3)$, Doun Penh $(n=1)$ (Figure 3$)$. Typhoid fever cases were more dispersed: 19 of 31 were from Phnom Penh, but distributed in small numbers among a large number of districts.

Preliminary susceptibility data based on laboratory files revealed that only 1 of $71(1.4 \%)$ S. Paratyphi A isolates was resistant to ampicillin, none to sulphamethoxazole-trimethoprim (SMX-TMP) and three (4.2\%) displayed nalidixic acid resistance.

In contrast, 14 out of $31 \mathrm{~S}$. Typhi isolates were ampicillin resistant, 11 were SMX-TMP resistant and all but two isolates were nalidixic acid resistant. Six isolates displayed high level ciprofloxacin resistance. Results of minimal inhibitory concentrations for ciprofloxacin are pending.

Empiric treatment included most often ceftriaxone followed by oral ciprofloxacin. So far, there was neither in-hospital mortality, nor relapse recorded.

\section{Discussion and conclusion}

Between January 2011 and August 2013, and particularly in the first eight months of 2013, we noted a remarkable increase in paratyphoid fever mainly among young adults treated in our hospital and clinics in Phnom Penh. Compared to the recovery of only two $S$. Paratyphi A isolates during the surveillance period 2007-2010 [9], this represents a 44-fold increase, while the monthly rate of blood cultures remained constant around 150-200. In June we observed a temporary drop in cases, for which we do not have a conclusive explanation, although it may be possible that cases coincidentally visited other healthcare facilities (mostly without culture facilities) within the metropolitan area.

Although it was not possible in the present setting to calculate population-based incidence data, the clustering in time and place of the recent $S$. Paratyphi A cases is of concern and suggests the implication of a common and persistent source. This could be either a continuing disseminating source (i.e. water) or a continuing point source such as a food vehicle. Consumption of food from street vendors has been found an independent risk factor for acquisition of paratyphoid fever in other Asian countries e.g. Nepal and Indonesia $[13,14]$. Of note, we observed also a two-fold increase of infections due to $S$. Typhi. Given the fact that predominantly adolescents and adults visit our hospital, more data on the possible spread of paratyphoid fever among Cambodian children are certainly needed.

Our findings coincide with the observation of increased numbers of $S$. Paratyphi A infection among European travellers from France, Germany, the Netherlands, New Zealand, Norway and the United Kingdom returning from Cambodia as communicated in a rapid risk 
assessment by the European Centre for Disease Prevention and Control (ECDC) on 4 September [1]. Cases from France are described in detail by Tourdjman and colleagues in this issue of Eurosurveillance [15].

Of note, most S. Paratyphi A isolated in Cambodia between 2011 and 2013 and from travellers in 2013, displayed low resistance levels for most commonly used antibiotics in contrast with S. Typhi and other Gramnegative pathogens in Cambodia studied between 2007 and 2010 [7]. To enable a more refined resistance description, we plan further batch-tested determination of the minimal inhibitory concentrations for ciprofloxacin, nalidixic acid and azithromycin amongst other antibiotics.

Further in-depth epidemiological research and a comparative analysis of clonal relationships between the Cambodian and European isolates are warranted to identify the source of the outbreak. Both findings, those in Cambodian citizens and European travellers, suggest that the 'hotspot' of this outbreak may be located in Phnom Penh, home to over a million inhabitants and a major gateway for visitors to the country.

Our findings were shared with the Ministry of Health of Cambodia, to allow the initiation of in-depth epidemiological investigations in order to organise the required public health measures. Cambodia, like many other low- and middle-income countries, is still building up its microbiological diagnostic capacity; across the country, less than 15 microbiology laboratories are in function [8]. In these settings, even small-scale clinical laboratories, such as the one in our hospital, may play an important role as 'sentinel' for emerging pathogens and resistance patterns.

\section{Acknowledgements}

This work was supported by Project 2.08 of the third Framework Agreement between the Belgian Directorate General of Development Cooperation (http://diplomatie. belgium.be/en/policy/development_cooperation/) and the Institute of Tropical Medicine, Antwerp (Belgium).

\section{Conflict of interest}

None declared.

\section{Authors' contributions}

Conceived and designed the study: JJ, EV, BDS.

Collected microbiological data: CK,DS,BDS.

Collected clinical and epidemiological data: TP, CHV, KL.

Analysed data: EV, BDS, TP, JJ, JvG.

Wrote the paper: EV, JJ, JVG, TP,ST.
References

1. European Centre for Disease Prevention and Control (ECDC) Rapid Risk Assesment. Unusual increase in reported cases of paratyphoid A fever among travellers returning from Cambodia. 4 September 2013. Stockholm: ECDC. Available from: http://www.ecdc.europa.eu/en/publications/Publications/ paratyphoid-fever-cambodia-rapid-risk-assessment.pdf

2. Crump JA, Mintz ED. Global trends in typhoid and paratyphoid Fever. Clin Infect Dis. 2010; 50(2):241-6. http://dx.doi. org/10.1086/649541. PMid:20014951. PMCid:PMC2798017.

3. Parry CM, Threlfall EJ. Antimicrobial resistance in typhoidal and nontyphoidal salmonellae. Curr Opin Infect Dis. 2008; 21(5):531-8. http://dx.doi.org/10.1097/QCO.ob013e3283of453a. PMid:18725804.

4. Ochiai RL, Wang X, von Seidlein L, Yang J, Bhutta ZA, Bhattacharya SK, et al. Salmonella paratyphi A rates, Asia. Emerg Infect Dis. 2005; 11(11):1764-6. http:// dx.doi.org/10.3201/eid1111.050168. PMid:16318734. PMCid:PMC3367370.

5. Kasper MR, Sokhal B, Blair PJ, Wierzba TF, Putnam SD. Emergence of multidrug-resistant Salmonella enterica serovar Typhi with reduced susceptibility to fluoroquinolones in Cambodia. Diagn Microbiol Infect Dis. 2010; 66(2):207-9. http://dx.doi.org/10.1016/j.diagmicrobio.2009.09.002. PMid:19800753.

6. Stoesser N, Moore CE, Pocock JM, An KP, Emary K, Carter M, et al. Pediatric bloodstream infections in Cambodia, 2007 to 2011. Pediatr Infect Dis J. 2013;32(7):e272-6. http://dx.doi. org/10.1097/INF.ob013e31828ba7c6. PMid:23838788.

7. Vlieghe ER, Phe T, De Smet B, Veng HC, Kham C, Lim K, et al. Bloodstream infection among adults in Phnom Penh, Cambodia: key pathogens and resistance patterns. PLoS One. 2013;8(3):e59775. http://dx.doi.org/10.1371/journal. pone.0059775. PMid:23555777. PMCid:PMC3612098.

8. Vlieghe ER, Sary S, Lim K, Sivuthy C, Phe T, Parry C, et al. First National Workshop on Antibiotic Resistance in Cambodia: Phnom Penh, Cambodia, 16-18 November 2011. Journal of Global Antimicrobial Resistance. 2013;1(1):31-4. http://dx.doi. org/10.1016/j.jgar.2013.01.007

9. Vlieghe ER, Phe T, De Smet B, Veng CH, Kham C, Bertrand S, et al. Azithromycin and ciprofloxacin resistance in salmonella bloodstream infections in cambodian adults. PLoS Negl Trop Dis. 2012;6(12): e1933.

10. Levy MM, Fink MP, Marshall IC, Abraham E, Angus D, Cook D, et al. 2001 SCCM/ESICM/ACCP/ATS/SIS International Sepsis Definitions Conference. Crit Care Med. 2003;29(4):530-8.

11. Clinical and Laboratory Standards Institute (CLSI). CLSI M100-S23. Performance Standards for Antimicrobial Susceptibility Testing; Twenty-third Informational Supplement. Wayne, PA: CLSI; 1 Jan 2013.

12. Grimont PA, Weill F.X. Antigenic formulae of the Salmonella serovars. 9th edition. 2007. Paris: WHO Collaborating Centre for Reference and Research on Salmonella, Institut Pasteur. Available from: http://www.pasteur.fr/ip/portal/action/ WebdriveActionEvent/oid/015-000036-089

13. Karkey A, Thompson CN, Tran Vu Thieu N, Dongol S, Le Thi Phuong T, Voong Vinh $\mathrm{P}$, et al. Differential epidemiology of salmonella typhi and paratyphi a in kathmandu, Nepal: a matched case control investigation in a highly endemic enteric Fever setting. PLoS Negl Trop Dis. 2013; 7(8):e2391. http:// dx.doi.org/10.1371/journal.pntd.0002391. PMid:23991240. PMCid:PMC3749961.

14. Vollaard AM, Ali S, van Asten HA, Widjaja S, Visser LG, Surjadi $C$, et al. Risk factors for typhoid and paratyphoid fever in Jakarta, Indonesia. JAMA. 2004; 291(21):2607-15. http://dx.doi. org/10.1001/jama.291.21.2607. PMid:15173152.

15. Tourdjman M, Le Hello S, Gossner C, Delmas G, Tubiana $S$, Fabre L, et al. Unusual increase in reported cases of Paratyphoid A fever among travellers returning from Cambodia, January to September 2013. Euro Surveill. 2013;18(39):pii=20594. Available from: http://www. eurosurveillance.org/ViewArticle.aspx?Articleld=20594 